\title{
Assessment of pyrogenic contaminations with validated human whole-blood assay
}

\author{
Mardas Daneshian ${ }^{1}$, Sonja von Aulock ${ }^{1,2}$ \& Thomas Hartung ${ }^{1,3}$ \\ ${ }^{1}$ Biochemical Pharmacology, University of Konstanz, Konstanz, Germany. ${ }^{2}$ Zukunftskolleg, University of Konstanz, Konstanz, Germany. ${ }^{3}$ Doerenkamp-Zbinden-Chair \\ for Evidence-based Toxicology, Bloomberg School of Public Health, Johns Hopkins University, Baltimore, Maryland, USA. Correspondence should be addressed to T.H. \\ (thartung@jhsph.edu).
}

\begin{abstract}
We present an internationally validated protocol for the evaluation of pyrogenic contaminations using human whole blood. In the in vitro pyrogen test (IPT) the sample is incubated with fresh or cryopreserved human whole blood, and the proinflammatory cytokine interleukin-1 $\beta$ (IL-1 $\beta$ ) is detected by enzyme-linked immunosorbent assay (ELISA). In addition to detecting pyrogenic contaminations in aqueous samples, e.g., parenteral drugs; adaptations allow the assessment of lipidic, toxic or immunomodulatory substances; detection of low-grade contaminations in large-volume parenterals, e.g., dialysis water and fluids; pyrogenicity assessment of solid materials, e.g., medical devices; and evaluation of airborne pyrogenic burden. In contrast to the rabbit pyrogen test and the limulus amoebocyte lysate (LAL) test, it requires no components of animal origin. In comparison with the LAL, it also detects nonlipopolysaccharide pyrogens. In comparison with other monocyte activation tests it requires no cell preparation steps or cell culture facilities. The procedure takes 21-35 h to complete.
\end{abstract}

\section{INTRODUCTION}

Pyrogenic (pyros is Greek for fire) contaminations pose a major safety concern for patients because their introduction into the human body, e.g., via parenterals, cell therapies or medical devices may cause severe reactions ranging from fever to shock and death. Known pyrogens are of microbial origin, but their presence does not correlate with colony-forming units, i.e., number of viable microorganisms, or with the presence of microbial genetic material. Examples of known pyrogens include lipopolysaccharides (LPS), i.e., well-described, highly potent endotoxins of Gram-negative bacteria; lipoteichoic acid (LTA) ${ }^{1}$, lipopeptides and peptidoglycan ${ }^{2}$ from Gram-positive bacteria; exotoxins ${ }^{3}$; enterotoxins ${ }^{4,5}$; viruses $^{6}$; and fungal components $s^{7,8}$.

\section{Development of the protocol}

In mammals the exposition of inner tissues to pyrogens leads to the secretion of the so-called endogenous pyrogens by immune cells, i.e., blood monocytes and tissue macrophages ${ }^{9}$, which when injected into mammals induce fever reactions. The most potent endogenous pyrogen is the proinflammatory cytokine interleukin (IL)-1 $\beta$ (refs. 10,11), which binds to receptors on the blood side of the organum vasculosum laminae terminalis, one of the circumventricular organs of the brain, and initiates the expression of the enzyme cyclooxygenase-2 (COX-2) ${ }^{12,13}$, which converts arachidonic acid to prostaglandin $(\mathrm{PG}) \mathrm{E}_{2}$. $\mathrm{PGE}_{2}$ then mediates an increase in temperature ${ }^{14}$. Mice deficient in COX-2 do not develop fever in response to injection of IL- $1 \beta$ or LPS ${ }^{15-17}$.

Our basic idea that led to the development of the in vitro pyrogen test (IPT) was to use human immune cells to detect exogenous pyrogens by measuring the release of an endogenous pyrogen ${ }^{18}$. Diluted fresh or cryopreserved blood is incubated for $10-24 \mathrm{~h}$ at $37^{\circ} \mathrm{C}$ with the test samples, spiked test samples, endotoxin controls and negative controls. The blood monocytes produce proinflammatory cytokines in response to any pyrogen present in a concentration-dependent manner and the IL- $1 \beta$ produced is measured by enzyme-linked immunosorbent assay (ELISA).

Human whole blood containing monocytes was chosen as an accessible source of primary human immune cells. The advantage of using whole blood instead of isolated cells, such as blood monocytes, is that all the immune cell types and serum components are present in their natural composition, and sources of contamination or preactivation of the cells are reduced as no isolation procedures are required.

Interleukin- $1 \beta$ was chosen as a readout parameter as it is consistently released in a concentration-dependent manner by blood from different donors ${ }^{19}$ upon exposure to the same minimal pyrogenic stimulation and can be readily measured by an ELISA. The sandwich ELISA is a highly specific and sensitive immunological assay that can detect antigens in the low $\mathrm{pg} \mathrm{ml}^{-1}$ range. The use of two antibodies, usually one monoclonal and one polyclonal antibody preparation, ensures high specificity, while linking the secondary antibody, directly or via biotin-streptavidin binding, to the enzyme horseradish peroxidase that metabolizes tetramethylbenzidine (TMB) ensures a high level of sensitivity.

The IPT thus poses a physiologically relevant human test system for the assessment of pyrogenic contaminations. It has successfully undergone an international validation process ${ }^{20,21}$ for both fresh and cryopreserved blood, and will be included in the European Pharmacopoeia in 2010 as announced by the European Directorate for the Quality of Medicines \& Health Care (www.edqm.eu/medias/ fichiers/133rd_Session_of_the_Eu.pdf). A similar statement by the United States Food and Drug Administration is pending. The IPT test will help to significantly reduce the use of animals for research and safety assessments in cosmetic and pharmaceutical industry. The methodology of IPT using either fresh or cryopreserved blood as well as its adaptations described herein are protected for commercial use by international patents $\mathrm{s}^{22-24}$.

\section{Applications of the method}

We have adapted the basic protocol of IPT, originally developed for the detection of pyrogens in aqueous liquid samples such as parenteral medications using fresh human whole blood, to a variety of different specific requirements. These adaptations include the development of cryopreserved human whole blood as an alternative to fresh human whole blood ${ }^{25}$, the assessment of the pyrogenicity 


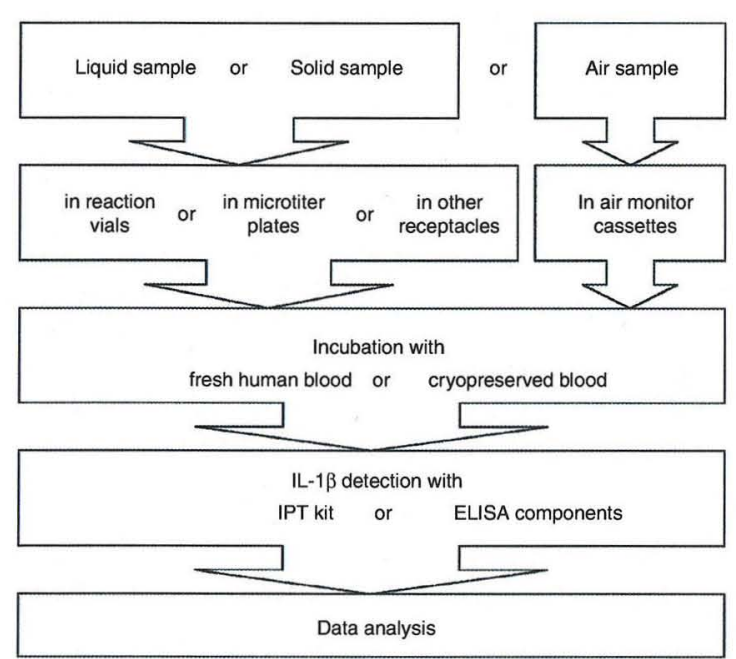

Figure 1 | Overview of protocol options.

of solid materials, such as medical devices ${ }^{26,27}$, and the evaluation of airborne pyrogenic burden ${ }^{28}$, all of which are detailed herein. The assay opens up new avenues for the control of cell therapies (from blood infusions to stem cells), medical devices and topical applications of substances with specific concerns (intrathecal injections, eye drops and baby food). It allows the assessment of laboratory reagents (e.g., recombinant proteins) and equipment (e.g., cell culture materials) for pyrogenic contaminations.

Other applications for more specialized requirements are described elsewhere. They include a specific protocol for testing lipidic substances and lipid-enclosed drugs that may interfere with pyrogen detection ${ }^{29}$. Some drugs may be cytotoxic or immunomodulatory and thus interfere with the whole-blood test independent of their containing possible pyrogenic contaminations. We have overcome this problem by collecting pyrogens from such samples onto bead-immobilized human serum albumin (HSA-beads) ${ }^{30,31}$. This allows the separation of pyrogens from the interfering drug by washing the beads and subjecting them to IPT. Similarly, the HSA-beads allow the accumulation of pyrogens from water samples or samples of dialysis fluids, where even very low-grade concentrations of pyrogens may be relevant, as for example a dialysis patient is exposed to many liters of dialysis fluids per session.

\section{Comparison with other methods}

Until now, pyrogenic contamination of parenterals has been assessed by animal testing, i.e., the rabbit pyrogen test ${ }^{32,33}$, or by employing the limulus amoebocyte lysate (LAL) test. Both tests carry essential limitations: in the rabbit test, next to ethical concerns, animal handling easily gives false-positive or negative results ${ }^{34}$, humans differ in their sensitivity to pyrogens from rabbits, and rabbits cannot be employed to test a range of modern pharmaceutical products, especially biologicals, cancer drugs and immunomodulatory drugs, owing to interference effects ${ }^{35}$. It should be noted that the rabbit assay, which was adopted in 1940, has never been formally validated, and no conclusive data for the detection of pyrogens other than LPS are available. The LAL, which employs the blood of horseshoe crab Limulus polyphemus, drawn from living crabs, detects only LPS and does not reflect the different potencies of LPS from different bacterial species observed in humans $s^{36,37}$. It also gives false-positive reactions in the presence of glucans and a number of herbal preparations ${ }^{35}$.
Further in vitro pyrogen tests using other cell populations, i.e., peripheral blood mononuclear cells or the cell line Monomac6 were internationally validated in parallel with the test described here ${ }^{20,21,38}$. In contrast to these, the whole-blood assay requires no cell preparation procedures that are more time consuming and are prone to preactivation of cells, and no cell culture, which requires special facilities and is available as a ready-to-use, qualitycontrolled kit, thereby minimizing the time to set up and validate the assay.

\section{Experimental design}

Sample types. Sterile, pyrogen-free materials must be used for all steps excluding the ELISA procedure. It is recommended that the whole-blood incubation steps be carried out under a laminar flow bench to avoid contaminations. An overview of the different protocol options developed for specific applications and to suit users' requirements is given in Figure 1. The type of sample, whether liquid, solid or air determines the whole-blood incubation format. With regard to liquid samples and some solid samples, depending on their dimensions, the whole-blood incubation may be carried out in $1.5 \mathrm{ml}$ reaction vials or in microtiter plates. Microtiter plates are recommended where the sample volume is limiting, however, they must be placed in an incubator. In contrast, $1.5 \mathrm{ml}$ reaction vials can alternatively be inserted into a thermoblock if an incubator is not accessible. For solid samples that are too large for plates or vials, a pyrogen-free incubation vessel must be identified in which the sample can be completely submerged for whole-blood incubation, e.g., $50 \mathrm{ml}$ falcons or depyrogenized glassware or metal vessels may be suitable. Glassware can be depyrogenized by incubation with $0.5 \mathrm{M} \mathrm{NaOH}$ for $30 \mathrm{~min}$, followed by extensive washing with purified water (e.g., Milli-Q); the pyrogenic activity of water should be tested before use. Alternatively, heat-stable glassware or metal vessels may be covered with aluminum foil and submitted to dry sterilization at $250^{\circ} \mathrm{C}$ for $3 \mathrm{~h}$, followed by extensive washing with purified water (e.g., Milli-Q). Determination of the pyrogenic burden in air requires the collection of airborne particles on a pyrogen-free, biocompatible membrane to allow interaction of the blood with the particles (see Box 1 for details). A resealable air monitor cassette containing a polytetrafluoroethylene (PTFE) membrane with a pore diameter of $5 \mu \mathrm{m}$ has proven useful for this. Sealed air monitor cassettes may be stored dry at $4{ }^{\circ} \mathrm{C}$ for up to $7 \mathrm{~d}$ after sampling.

Aqueous liquid samples may need to be employed in different dilutions (e.g., a 1:10 dilution series) to exclude interference (see below) or to allow a semi-quantitative readout (see below). A semi-quantitative assessment of the pyrogenic burden of solid samples is only possible if the sample can be employed in different quantities, e.g., fibers and beads. Otherwise only a rough estimation is possible (see below). For the assessment of pyrogenic activity in air samples, it is recommended to pump a broad range of air volumes through different air monitor cassettes (e.g., 1, 3, 10, 30,100 and 300 liters) to allow a semi-quantitative assessment (see below) of the pyrogenic burden.

Use of fresh blood or cryopreserved blood. Fresh human whole blood should be obtained from a healthy and nonallergic donor who has not taken any medication for at least $14 \mathrm{~d}$. The blood is drawn by venipuncture into sterile, heparinized vials. Blood may only be drawn by a trained physician or nurse in accordance 


\section{BOX 1 | MEASUREMENT OF AIRBORNE PYROGENS}

(i) Collect airborne pyrogens by drawing different amounts of air through the air monitor cassettes at a flow rate of 1 liter min ${ }^{-1}$. Pump a broad range of air volumes through different air monitor cassettes (e.g., 1, 3, 10, 30, 100 and 300 liters).

PAUSE POINT Monitors may be stored dry at $4{ }^{\circ} \mathrm{C}$ for $4-7 \mathrm{~d}$.

(ii) Set up the whole blood or cryopreserved blood incubations as described in step 4 (A) and (B), respectively, with a final volume of 3,600 $\mu$ l. See Tables 2 and 3.

(iii) Seal the monitors with the pins provided and mix by gentle inversion.

(iv) Incubate the samples as described in Step 5.

(v) Transfer $>300 \mu \mathrm{l}$ of samples to $1.5 \mathrm{ml}$ tubes or for storage transfer $150 \mu \mathrm{l}$ aliquots to round bottom microtiter plates; seal with a plastic cover.

PAUSE POINT Samples can be stored at $-20^{\circ} \mathrm{C}$ for no longer than 4 weeks or at $-80^{\circ} \mathrm{C}$ until required.

(vi) Carry out the ELISA in Steps 8-17 using $50 \mu$ l of samples.

(vii) Analyze the data as outlined in Steps 18-21.

with the legal requirements of the country and guidelines of the institution. Determination of a normal white blood cell count, e.g., by blood smear and microscopic evaluation or by using either an electronic cell counter or image analysis instrument, is recommended to exclude acute infection. The blood must be used for incubation within $4 \mathrm{~h}$ of being drawn, as the sensitivity of the blood to pyrogens decreases after this time. During this time it may be stored at room temperature $\left(15-30^{\circ} \mathrm{C}\right)$.

The choice between fresh blood and cryopreserved blood is determined by a laboratory's accessibility to fresh human blood within $4 \mathrm{~h}$ of starting the whole-blood incubation step and the possibility to store cryopreserved blood at $-70^{\circ} \mathrm{C}$ or in liquid nitrogen. Cryopreserved blood can be produced ${ }^{25}$ or obtained in large lots from a pool of donations by different healthy donors, and the batch can be pretested for HIV or Hepatitis B antibodies as an added safety precaution. Cryopreserved blood must be incubated in a $5 \% \mathrm{CO}_{2}$ atmosphere to ensure cytokine release on pyrogenic stimulation.

The total volume of blood required is determined by the number of samples and controls, and the volume of incubations, e.g., $20 \mu \mathrm{l}$ per well for microtiter plates, $100 \mu \mathrm{l}$ per reaction vial or $300 \mu \mathrm{l}$ per air monitor cassette for airborne samples. Fresh blood is diluted in clinical-grade saline $(150 \mathrm{mM})$ to a final dilution of $1: 12$. Cryopreserved blood is already diluted $1: 2$ by the cryopreservation procedure and is further diluted 1:6 in RPMI 1640 to a final dilution of 1:12. The dilution with RPMI 1640 must occur immediately after thawing for $15 \mathrm{~min}$ at $37^{\circ} \mathrm{C}$ to avoid toxic effects of the cryopreservative DMSO. The dilution of 1:12 results in a high release of cytokines using a minimal amount of blood. A recent report demonstrated that the cytokine inhibitor $\alpha-1$-antitrypsin is no longer active at this dilution, which explains why the cytokine release is higher and thus more robustly detectable in diluted samples ${ }^{39}$.

Detection of IL- $1 \beta$ release. The levels of IL-1 $\beta$ released in the course of the whole-blood incubation are determined by ELISA. For diagnostic, clinical or commercial purposes the qualityassured IPT kit, which contains all reagents required for the wholeblood incubation apart from the blood itself, is recommended. For research purposes, the cheaper option of an IL-1 $\beta$ ELISA kit or respective antibody pairs may be chosen instead. An IL-1 $\beta$ ELISA kit generally contains all ELISA components including the antibodies, recombinant IL- $1 \beta$ standard (not required for IPT), microtiter plate, buffers and other reagents as well as a detailed protocol, which also gives the sensitivity and specificity of the assay. Antibody pairs are pretested for compatibility in the respec- tive ELISA, and a recommendation for the concentration to be used is usually given in the data sheet by the manufacturer. The other ELISA components can be bought in bulk, and the buffers can be mixed using standard laboratory chemicals. The ELISA must then be set up, optimized and validated. The sensitivity is determined using recombinant IL-1 $\beta$ cytokine and must reach down to the low $\mathrm{pg} \mathrm{ml}^{-1}$ range to allow IPT testing. Setting up an ELISA in this way requires some experience in immunological techniques but reduces the cost per ELISA substantially in comparison with the IPT kit or the ELISA kit if the assay is carried out regularly. New batches of components must always be validated against the former batches to ensure consistency of results.

The ELISA plate is coated with the primary antibody. The IL-1 $\beta$ in the sample is then sandwiched between the primary coat antibody and a secondary horseradish peroxidase-labeled detection antibody or a biotinylated detection antibody. In the latter case, a streptavidin-linked horseradish peroxidase is added after washing. The unbound material is removed by washing. The peroxidase metabolizes the substrate $3,3^{\prime}, 5,5^{\prime}-\mathrm{TMB}$. The reaction is stopped with hydrochloric or sulfuric acid, and the optical density (OD) is measured at $450 \mathrm{~nm}$ against a reference wavelength of 600 to $690 \mathrm{~nm}$. No IL-1 $\beta$ reference material is required to quantify IL- $1 \beta$ protein as the ELISA OD readout is compared with that of the endotoxin controls. It is to be noted that various other cytokines (e.g., IL-6, TNF and IL-8) and PGE $_{2}$ and assays on protein or mRNA level have been shown to give similar results as the IL- $1 \beta$ ELISA but require in-case validation against the standard protocol ${ }^{18}$.

Design of an incubation plan. The design of an incubation plan is essential before the initiation of the experiment. Each incubation setup should be composed of endotoxin controls (0.5 EU $\mathrm{ml}^{-1}$ is necessary for qualitative assessment; a range of endotoxin controls, e.g., $0.25,0.5,1.0,2.5$ and $5.0 \mathrm{EU} \mathrm{ml}^{-1}$ is required for semi-quantitative assessment), a negative control (saline for fresh blood or RPMI 1640 for cryopreserved blood), samples and respective spiked samples (spiked with $0.5 \mathrm{EU} \mathrm{ml}^{-1}$ of the endotoxin control). Spiked samples are only required for interference testing in new samples. Data should be collected in quadruplicate. Ideally, the incubation plan should already correspond to the given requirements of the respective ELISA reader software to allow easy transfer of samples after the whole-blood assay onto the ELISA plates. Table 1 presents a recommended incubation plan. The number of samples and spiked samples will vary depending on the experiment. 
Endotoxin controls. The endotoxin controls are set up with the international World Health Organization (WHO) reference standard from Escherichia coli O113:H10 (ref. 40) or another LPS that has been calibrated against this WHO reference standard by LAL assay, e.g., control standard endotoxin (CSE) or the LPS from E. coli O111:B4 included in the IPT kit. As LPS from different species may differ in potency and preparations from the same species may vary in potency between batches, the calibration against the WHO standard ensures that the same stimulus potency is used in every assay, thus ensuring comparability of results. One concentration of the endotoxin controls, i.e., $0.5 \mathrm{EU} \mathrm{ml}^{-1}$, is also employed to prepare spiked samples for an interference test, which is required for any new sample types or drug formulations (see below). The spike is allowed to interact with the sample for $2 \mathrm{~h}$ at room temperature, i.e., $15-30^{\circ} \mathrm{C}$ before incubation with human whole blood.

Statistical analysis. All samples are assayed in quadruplicates. This allows outlier analysis using the methods described by Dixon or Grubbs $^{41,42}$

Validity of the assay. Two criteria must be fulfilled for the assay to be valid. First, the mean OD of the negative controls must lie at or below 100 mean OD. Second, the mean OD of $0.5 \mathrm{EU} \mathrm{ml}^{-1}$ control must be greater than 1.6 times the mean OD of the negative controls. The endotoxin control at a concentration of $0.5 \mathrm{EU} \mathrm{ml}^{-1}$ corresponds to $50 \mathrm{pg} \mathrm{ml}^{-1}$ of the WHO international reference standard from E. coli $\mathrm{O} 113: \mathrm{H} 10$ and is the threshold endotoxin concentration that causes fever in $50 \%$ of animals of the most sensitive rabbit strains. This threshold was confirmed by a study carried out at the Paul-Ehrlich Institute in 2005, which analyzed 171 rabbits $^{43}$.

Interference test. To exclude putative interferences of test substances with the activity of blood cells, e.g., toxicity or immunomodulation, new sample types or drug formulations should be spiked with an endotoxin control $\left(0.5 \mathrm{EU} \mathrm{ml}^{-1}\right)$ in parallel to the incubation with the unspiked sample. The mean OD of the spiked sample replicates must lie within a $50-200 \%$ range of the $0.5 \mathrm{EU} \mathrm{ml}^{-1}$ concentration of the endotoxin control. If this is not the case, then the sample is interfering with the assay and the experiment must be repeated using a diluted sample and spiked once again until the interference is excluded. However, the maximum valid dilution (MVD) or minimal valid concentration (MVC) may not be overstepped. These are calculated figures that indicate the degree to which a product may be diluted to overcome interference before the effect of dilution exceeds the ability of the test method to detect pyrogens in the original preparation. The MVD is usually applied to preparations already in liquid form, wherein the dose is administered per $\mathrm{ml}$ and the endotoxin limit is expressed as $\mathrm{EU} \mathrm{ml} \mathrm{ml}^{-1}$. The MVC is applied to those preparations wherein the endotoxin limit is expressed as $\mathrm{EU} \mathrm{mg}^{-1}$ and the dose is expressed as $\mathrm{mg} \mathrm{kg}^{-1}$ body weight. If interference cannot be excluded before MVD or MVC is reached, other assay variants may be helpful ${ }^{30,31}$. Interference must only be excluded for each new sample, i.e., it does not have to be repeated for every batch of similar preparation.

Estimation of the sample pyrogenic activity. Samples that induce the release of values of IL- $1 \beta$ higher than the positive control sample containing $0.5 \mathrm{EU} \mathrm{ml}^{-1}$ are considered positive for pyrogenic contamination (qualitative assessment). The IPT also detects the activity of pyrogens other than LPS, e.g., LTA, exotoxins and fungal components, without identifying the pyrogen type or pyrogen mixture composition. The concentration response curves of different pyrogens do not necessarily run in parallel. Therefore, the readout of IPT is generally qualitative, i.e., the sample is positive or negative in comparison with $0.5 \mathrm{EU} \mathrm{ml}^{-1}$ endotoxin control.

For a semi-quantitative assessment of the pyrogenic activity using liquid samples, a limit dilution may be carried out, i.e., samples are employed in different dilutions (e.g., a series of 1:10 dilutions) and the dilution that results in IL-1 $\beta$ release that is comparable with $0.5 \mathrm{EU} \mathrm{ml}^{-1}$ endotoxin control is used to calculate the endotoxin contamination in the undiluted sample. This estimation is given in endotoxin-equivalent units (EEUs) in consideration of the fact that non-LPS pyrogens may be contributing to the pyrogenic activity. Similarly, a semi-quantitative assessment of the pyrogenic burden in air samples may be given in EEU by comparison of the IL- $1 \beta$ induction in the monitors sampled with different volumes of air. Again, the sample inducing IL-1 $\beta$ release comparable with $0.5 \mathrm{EU} \mathrm{ml}^{-1}$ endotoxin control should be used to calculate the total EEU in the

TABLE 1 | Example of an incubation plan.

\begin{tabular}{|c|c|c|c|c|c|c|c|c|c|c|c|c|}
\hline & 1 & 2 & 3 & 4 & 5 & 6 & 7 & 8 & 9 & 10 & 11 & 12 \\
\hline A & EC 2.5 & EC 0.5 & EC 0.12 & P01 & P02 & P03 & P04 & P05 & P06 & P07 & P08 & P09 \\
\hline B & EC 2.5 & EC 0.5 & EC 0.12 & P01 & P02 & P03 & P04 & P05 & P06 & P07 & P08 & P09 \\
\hline C & EC 2.5 & EC 0.5 & EC 0.12 & P01 & P02 & P03 & P04 & P05 & P06 & P07 & P08 & P09 \\
\hline D & EC 2.5 & EC 0.5 & EC 0.12 & P01 & P02 & P03 & P04 & P05 & P06 & P07 & P08 & P09 \\
\hline$E$ & EC 1.0 & EC 0.25 & EC 0.0 & $\mathrm{P}^{\prime \prime} 1^{\prime}$ & $\mathrm{P}^{\prime} 2^{\prime}$ & $\mathrm{P}^{\prime \prime}$ & P04' & $\mathrm{PO5}^{\prime}$ & P06' & P07' & P08' & PO9' \\
\hline$F$ & EC 1.0 & EC 0.25 & EC 0.0 & $\mathrm{P} 01^{\prime}$ & $\mathrm{P}^{\prime} 2^{\prime}$ & $\mathrm{P}^{\prime \prime} 3^{\prime}$ & P04' & P05' & P06' & P07' & P08' & PO9' \\
\hline G & EC 1.0 & EC 0.25 & EC 0.0 & $\mathrm{P}^{\prime} 1^{\prime}$ & $\mathrm{P}^{\prime \prime} 2^{\prime}$ & $\mathrm{P}^{\prime \prime}$ & $\mathrm{P}^{\prime \prime}$ & $\mathrm{POS}^{\prime}$ & $\mathrm{P} 06^{\prime}$ & P07' & P08' & POg' \\
\hline $\mathrm{H}$ & EC 1.0 & EC 0.25 & EC 0.0 & P01' & P02' & P03' & P04' & $\mathrm{P}^{\prime} 5^{\prime}$ & P06' & P07' & P08' & POg' \\
\hline
\end{tabular}

$\mathrm{EC}$, endotoxin control given in $\mathrm{EU} \mathrm{ml}^{-1}$. P01-P09, samples. $\mathrm{P}^{\prime} 1^{\prime}-\mathrm{PO9}^{\prime}$, samples spiked with $0.5 \mathrm{EU} \mathrm{ml}^{-1}$. 
respective volume of sampled air. For solid samples a similar semiquantitative assessment is possible if the samples can be employed in different quantities, e.g., fibers and beads. Otherwise only a rough estimation can be made by finding comparable IL- $1 \beta$ release in response with the sample and one of the endotoxin controls in the linear part of the concentration response curve. This rough estimation would not consider the different shapes of concentration response curves stemming from different types of pyrogens.
Timing. It is most efficient to set up the whole-blood incubation during one working day, leave it to incubate overnight and carry out the ELISA on the following day. Although some ELISA kits contain plates already coated with the primary antibody, other kits as well as ELISA's setup with antibody pairs will require the primary antibody to first be coated to the ELISA plate. This may be set up on the first day, leaving the coating process to occur overnight at $4{ }^{\circ} \mathrm{C}$ parallel to the whole blood incubation.

\section{MATERIALS}

\section{REAGENTS}

IPT using fresh human whole blood

- Human whole blood from healthy volunteers (stored for up to $4 \mathrm{~h}$ at room temperature)! CAUTION Human blood may transmit infectious diseases such as Hepatitis B or HIV. Use protective gloves, dispose of contaminated sharp waste in safety containers at the point of use, dispose of contaminated waste after autoclaving. I CAUTION Experiments with human subjects must comply with institutional and national guidelines.

IPT using cryopreserved human whole blood

- Cryopreserved blood prepared according to ${ }^{21}$ or obtained from commercial sources, e.g., Qualis Laboratorium $\mathrm{GmbH}$. Blood may be stored at $-70^{\circ} \mathrm{C}$ or in liquid nitrogen for at least 6 months I CAUTION Human blood may transmit infectious diseases such as Hepatitis B or HIV. Use protective gloves, dispose of the contaminated sharp waste in safety containers at the point of use, dispose of contaminated waste after autoclaving.

- Pyrogen-free RPMI 1640 (included in the IPT kit) (e.g., BioWhittaker Europe, Lonza, cat. no. BE12-702G/U1).

IPT for diagnostic, clinical or commercial purposes using the IPT kit

- Endosafe IPT kit (Charles River Laboratories International, cat. no. IPT100)

IPT for research purposes using ELISA components

- Clinical grade saline (e.g., B. Braun, cat. no. 2350556)

- Endotoxin control: the international WHO reference standard from E. coli O113:H10 (National Institute for Biological Standards and Control, cat. no. 94/580), or an LPS that has been calibrated against the WHO reference standard (e.g., CSE, Charles River Laboratories International, cat. no. E110)

- Antihuman IL-1 $\beta$ antibody pair, e.g., consisting of a coating antibody and a biotin-labeled detection antibody (e.g., MAB601 and BAF201, R\&D Systems)

- Streptavidin peroxidase (e.g., Streptavidin, horseradish peroxidase conjugate, Biosource, cat. no. SNN1004)

- $\mathrm{NaHCO}_{3}$ (e.g., Sigma, cat. no. S5761)

- $\mathrm{NaCl}$ (e.g., Sigma, cat. no. S3014)

- $\mathrm{KH}_{2} \mathrm{PO}_{4}$ (e.g., Sigma, cat. no. P9791)

- $\mathrm{Na}_{2} \mathrm{HPO}_{4}$ (e.g., Sigma, cat. no. S3264)

- KCl (e.g., Sigma, cat. no. P8041)

- Bovine serum albumin (e.g., PAA, cat. no. K45-001)

- Tween20 (e.g., Sigma, cat. no. P1379)

-3,3',5,5'-TMB substrate (e.g., Sigma, cat. no. T8665)! CAUTION Flammable. Toxic by inhalation, in contact with skin and if swallowed. Do not inhale the vapor. Avoid contact with eyes, skin and clothing. Avoid prolonged or repeated exposure. Do not use if skin is cut or scratched. Wash thoroughly after handling. Use in a fume hood.

- $1 \mathrm{M} \mathrm{H}_{2} \mathrm{SO}_{4}$ (e.g., Merck, cat. no. 100716) I CAUTION Causes severe burns. Do not inhale the vapor. Avoid contact with eyes, skin and clothing. Avoid prolonged or repeated exposure. Use in a fume hood.

\section{EQUIPMENT}

- Pyrogen-free reservoirs (e.g., $50 \mathrm{ml}$ falcon, Greiner Bio-one, cat. no. 210261)

- Sterile and pyrogen-free pipette tips (e.g., Biosphere Tips blue, Sarstedt, cat. nos. 70.760 .202 and 70.762 .200$)$
- Vortex mixer

- Round-bottomed microplates (e.g., PS Microplate, 96 well, U-shape; Greiner Bio-one, cat. no. 650101) with sealing tape (e.g., Nunc, cat. no. 232701)

- Pyrogen-free borosilicate test tubes (e.g., KairoSafe, cat. no. 1959000)

- Optional: Microplate mixer (e.g., MX4, Finepcr)

- Optional: Microplate washer

- Microplate reader capable of reading at $450 \mathrm{~nm}$ (reference filter in the range of 600-690 nm) (e.g., Sunrise,TECAN)

- Optional: Software package for data analysis and graphic representation (e.g., Prism 4.0, GraphPad )

IPT using fresh human whole blood

- Sterile, endotoxin-free, heparinized tubes for blood sampling (e.g., Sarstedt

S-Monovette $7.5 \mathrm{ml}, \mathrm{LH}$, Sarstedt, cat. no. 01.1608.001)

- Sterile needle set (Multifly Set $21 \mathrm{G}$ tubing 8", Sarstedt, cat. no. 85.1638.035)

- Antiseptic agent for dermal use (Freka-derm farblos, Fresenius Kabi, cat. no. 4928211)

- Incubator or thermoblock that can be adjusted to $37^{\circ} \mathrm{C} \pm 1{ }^{\circ} \mathrm{C}$

IPT using cryopreserved blood

- Incubator that can be adjusted to $37^{\circ} \mathrm{C} \pm 1{ }^{\circ} \mathrm{C}$ with $5 \% \mathrm{CO}_{2}$

IPT in reaction vials

- $1.5 \mathrm{ml}$ sealable, pyrogen-free reaction tubes (Eppendorf, cat. no. 0030 102.002)

IPT on microtiter plates

- 96-well flat-bottom tissue culture plates (TC-plate, Greiner Bio-one, cat. no. 655180 )

IPT using ELISA components

- Multisorp ELISA plates (F96 Maxisorp Nunc-Immunoplate, Nunc, cat. no. 442404)

IPT for airborne samples

- Air monitor cassettes with $5.0 \mu \mathrm{m}$ PTFE membranes (37 mm air monitor $5.0 \mu \mathrm{m}$ PTFE membrane, PALL Life Sciences, cat. no. 4269).

- Air sampling pump for low flows (1 liter $\mathrm{min}^{-1}$ ) (e.g., GSM, cat. no. SG400ex)

REAGENT SETUP

Endotoxin control Prepare a 2,000 EU ml-1 stock solution using clinical grade saline. Aliquots (e.g., $50 \mu \mathrm{l}$ ) can be stored at $-20^{\circ} \mathrm{C}$ for up to 6 months. Vortex stock solutions and all dilutions made from these for at least $60 \mathrm{~s}$ directly before use.

Coating buffer Prepare a solution of $0.1 \mathrm{M} \mathrm{NaHCO}_{3}$ in deionized water; can be stored at $-20^{\circ} \mathrm{C}$ for 6 months.

Blocking and dilution buffer Prepare a solution of $14 \mathrm{mM} \mathrm{NaCl}, 1.5 \mathrm{mM}$ $\mathrm{KH}_{2} \mathrm{PO}_{4}, 6.5 \mathrm{mM} \mathrm{Na}_{2} \mathrm{HPO}_{4}, 2.7 \mathrm{mM} \mathrm{KCl}$ in deionized water; adjust the $\mathrm{pH}$ to 7.0. Add $30 \mathrm{gl}^{-1}$ bovine serum albumin and stir until dissolved. Can be stored at $-20^{\circ} \mathrm{C}$ for 6 months. $\triangle$ CRITICAL Add bovine serum albumin after pH adjustment.

Washing buffer Prepare a solution of $14 \mathrm{mM} \mathrm{NaCl}, 1.5 \mathrm{mM} \mathrm{KH}_{2} \mathrm{PO}_{4}$, $6.5 \mathrm{mM} \mathrm{Na}_{2} \mathrm{HPO}_{4}, 2.7 \mathrm{mM} \mathrm{KCl}$ in deionized water; adjust the $\mathrm{pH}$ to 7.0 . Add $0.05 \%$ Tween 20 (vol/vol). Can be stored at $4{ }^{\circ} \mathrm{C}$ for 1 week. $\Delta$ CRITICAL Add Tween 20 after $\mathrm{pH}$ adjustment. 


\section{PROCEDURE}

\section{Incubation plan design TIMING 15 min}

1) Calculate the number of samples to be assayed. Include endotoxin controls (e.g., 0.25, 0.5, 1.0, 2.5 and 5.0 EU ml-1), a negative saline control (for fresh blood) or negative RPMI 1640 control (for cryopreserved blood), spiked samples $\left(0.5 \mathrm{EU} \mathrm{ml}^{-1}\right)$ if required and samples. Prepare each sample in quadruplicate. An example of an incubation plan is given in Table 1.

$\triangle$ CRITICAL STEP Only samples for which interference with the assay has not been previously excluded must also be included as spiked samples.

Prepare endotoxin controls TIMING 15 min

2) Dilute the endotoxin stock solution $\left(2,000 \mathrm{EU} \mathrm{ml}^{-1}\right)$ to the required endotoxin concentrations, e.g., $0.25,0.5,1.0,2.5$ and $5.0 \mathrm{EU} \mathrm{ml}^{-1}$, in clinical grade saline.

$\triangle$ CRITICAL STEP The endotoxin concentration $0.5 \mathrm{EU} \mathrm{ml}^{-1}$ must be included to allow qualitative assessment of pyrogenic contamination in the sample.

\section{Sample preparation TIMING 30 min}

3| Pyrogenic activity can be measured in option A liquid samples or option B solid materials. For measurement of pyrogenic activity in air samples see Box 1.

(A) Liquid samples

(i) Prepare the required liquid samples (e.g., dilutions if necessary) according to the incubation plan (see Table 1 for example).

(ii) The whole blood incubation may be carried out in $1.5 \mathrm{ml}$ sealable, pyrogen-free reaction tubes in a total volume of $1,200 \mu \mathrm{l}$ or 96 -well flat-bottom tissue culture plates in a total volume of $240 \mu \mathrm{l}$.

(B) Solid materials

(i) Place solid samples in sterile and pyrogen-free vials or receptacles in which they can be fully submerged with fluid.

\section{Whole-blood incubation TIMING 13-27 h}

4| Pyrogenic activity can be measured using option A fresh human whole blood or option B cryopreserved human whole blood.

I CAUTION All experiments using human blood must comply with the legal requirements of the country and guidelines of the institution. Human blood may transmit infectious diseases such as Hepatitis B or HIV. Use protective gloves, dispose of contaminated sharp waste in safety containers at the point of use, dispose of contaminated waste after autoclaving.

\section{(A) Fresh human whole blood}

(i) Determine the final volume of the assay by e.g., considering for liquid samples whether vials or a microtiter plate is being used (in which case, the final volume should be 1,200 $\mu \mathrm{l}$ and $240 \mu \mathrm{l}$, respectively) or whether solid samples are being used (in which case, the final volume should be adapted according to the requirements) (see Table 2).

(ii) Add nine parts clinical grade saline to the receptacles for the spiked samples and ten parts clinical grade saline to each other receptacle.

(iii) Add one part of the appropriate endotoxin control solution to the receptacles for endotoxin controls and spiked samples.

(iv) Add one part saline to the receptacles for negative controls.

(v) Add one part sample to the receptacles for samples and spiked samples.

(vi) Incubate at room temperature for $2 \mathrm{~h}$. Omit this step if the incubation does not include any spiked samples.

(vii) Resuspend the fresh blood (drawn less than $4 \mathrm{~h}$ before use) by gently inverting the closed heparinized vials.

(viii) Add one part fresh human whole blood to each receptacle to reach the required total volume.

(B) Cryopreserved human whole blood

(i) Determine the final volume of the assay by e.g., considering for liquid samples whether vials or a microtiter plate is being used (in which case, the final volume should be 1,200 $\mu \mathrm{l}$ and $240 \mu \mathrm{l}$, respectively) or whether solid samples are being used (in which case, the final volume should be adapted to your requirements) (see Table 3).

(ii) Add eight parts RPMI 1640 to the receptacles for spiked samples and nine parts clinical grade saline to each other receptacle. 
TABLE 2 | Setup of whole blood incubation for fresh human whole blood.

\begin{tabular}{|c|c|c|c|c|}
\hline & Negative control & Endotoxin control & Sample & Spiked sample \\
\hline \multicolumn{5}{|l|}{ Vials $^{\mathrm{a}}$} \\
\hline Saline & 11,00 & 1,000 & 1,000 & 900 \\
\hline Endotoxin solution & 0 & 100 & 0 & 100 \\
\hline Sample & 0 & 0 & 100 & 100 \\
\hline Fresh blood & 100 & 100 & 100 & 100 \\
\hline Final volume & 1,200 & 1,200 & 1,200 & 1,200 \\
\hline \multicolumn{5}{|l|}{ Plates $^{\mathrm{a}}$} \\
\hline Saline & 220 & 200 & 200 & 180 \\
\hline Endotoxin solution & 0 & 20 & 0 & 20 \\
\hline Sample & 0 & 0 & 20 & 20 \\
\hline Fresh blood & 20 & 20 & 20 & 20 \\
\hline Final volume & 240 & 240 & 240 & 240 \\
\hline \multicolumn{5}{|l|}{ Other } \\
\hline Saline & 11 & 10 & 10 & 9 \\
\hline Endotoxin solution & 0 & 1 & 0 & 1 \\
\hline Sample & 0 & 0 & 1 & 1 \\
\hline Fresh blood & 1 & 1 & 1 & 1 \\
\hline \multicolumn{5}{|l|}{ Air monitor cassettes ${ }^{\mathrm{a}}$} \\
\hline Saline & 3,300 & 3,000 & 3,000 & 2,700 \\
\hline Endotoxin solution & 0 & 300 & 0 & 300 \\
\hline Sample & 0 & 0 & 300 & 300 \\
\hline Fresh blood & 300 & 300 & 300 & 300 \\
\hline Final volume & 3,600 & 3,600 & 3,600 & 3,600 \\
\hline
\end{tabular}

avalues given in $\mu$. VValues given in parts of the total volume for solid samples.

(iii) Add one part of the appropriate endotoxin control solution to the receptacles for endotoxin controls and spiked samples.

(iv) Add one part saline to the receptacles for negative controls.

(v) Add one part sample to the receptacles for samples or spiked samples.

(vi) Incubate at room temperature for $2 \mathrm{~h}$. Omit this step if incubation does not include any spiked samples.

(vii) Thaw the cryopreserved blood at $37{ }^{\circ} \mathrm{C}$ for a maximum period of $15 \mathrm{~min}$ to continue immediately with Step viii.

$\triangle$ CRITICAL STEP Use the thawed cryopreserved blood within 15 min to prevent the high concentration of DMSO from becoming toxic to the cells.

(viii) Resuspend the blood by gently inverting the closed vials. Combine blood from different vials into one pyrogen-free reservoir. Add two parts of cryopreserved human whole blood to each receptacle to reach the total incubation volume.

5| For samples assayed in vials mix the samples by closing the reaction vials and gently inverting the tubes a few times. For samples prepared in microtiter plates, mix by aspiring and dispensing five times. Change tips between each sample to avoid cross contamination. Cover the microtiter plate with a lid. For solid samples, mix the contents by gently inverting the receptacles by swinging them manually.

6| Incubate samples prepared with fresh blood in an incubator or a heating block for $10-24 \mathrm{~h}$ at $37^{\circ} \mathrm{C} \pm 1{ }^{\circ} \mathrm{C}$. Incubate samples prepared with cryopreserved blood in an incubator for $10-24 \mathrm{~h}$ at $37{ }^{\circ} \mathrm{C} \pm 1{ }^{\circ} \mathrm{C}$ and $5 \% \mathrm{CO}_{2}$. 
TABLE 3 | Setup of whole blood incubation for cryopreserved human whole blood.

\begin{tabular}{|c|c|c|c|c|}
\hline & Negative control & Endotoxin control & Sample & Spiked sample \\
\hline \multicolumn{5}{|l|}{ Vials $^{\mathrm{a}}$} \\
\hline RPMI 1640 & 1,000 & 900 & 900 & 800 \\
\hline Endotoxin solution & 0 & 100 & 0 & 100 \\
\hline Sample & 0 & 0 & 100 & 100 \\
\hline Cryopreserved blood & 200 & 200 & 200 & 200 \\
\hline Final volume & 1,200 & 1,200 & 1,200 & 1,200 \\
\hline \multicolumn{5}{|l|}{ Plates $^{\mathrm{a}}$} \\
\hline RPMI 1640 & 200 & 180 & 180 & 160 \\
\hline Endotoxin solution & 0 & 20 & 0 & 20 \\
\hline Sample & 0 & 0 & 20 & 20 \\
\hline Cryopreserved blood & 40 & 40 & 40 & 40 \\
\hline Final volume & 240 & 240 & 240 & 240 \\
\hline \multicolumn{5}{|l|}{ Other } \\
\hline RPMI 1640 & 10 & 9 & 9 & 8 \\
\hline Endotoxin solution & 0 & 1 & 0 & 1 \\
\hline Sample & 0 & 0 & 1 & 1 \\
\hline Cryopreserved blood & 2 & 2 & 2 & 2 \\
\hline \multicolumn{5}{|l|}{ Air monitor cassettes ${ }^{\mathrm{a}}$} \\
\hline Saline & 3,000 & 2,700 & 2,700 & 2,400 \\
\hline Endotoxin solution & 0 & 300 & 0 & 300 \\
\hline Sample & 0 & 0 & 300 & 300 \\
\hline Cryopreserved blood & 600 & 600 & 600 & 600 \\
\hline Final volume & 3,600 & 3,600 & 3,600 & 3,600 \\
\hline
\end{tabular}

values given in $\mu \mathrm{L}$. values given in parts of the total volume for solid samples.

Sample preparation for ELISA $\left(15-30^{\circ} \mathrm{C}\right)$

7| Mix the samples as described in Step 5. Aliquot the contents ( $\geq 300 \mu \mathrm{l})$ of the receptacles that contained solid samples into $1.5 \mathrm{ml}$ tubes. Samples in tubes can be used directly or be centrifuged for $2 \mathrm{~min}$ at $10,000 \mathrm{~g}$, room temperature and the cleared supernatant is collected. Samples can be used immediately for ELISA analysis or stored for later use or to repeat the assay. For storage, aliquot samples into microtiter plates and seal with a lid or plastic tape.

PAUSE POINT Sealed plates may be frozen at $-20^{\circ} \mathrm{C}$ for no longer than 4 weeks or at $-70^{\circ} \mathrm{C}$ until required.

Determining IL-1 $\beta$ content in samples by ELISA $\odot$ TIMING $20 \mathrm{~h}$

8| Coat a Multisorp ELISA plate with $50 \mu \mathrm{l}$ of primary antibody solution (e.g., MAB601 at $250 \mathrm{ng} \mathrm{ml}^{-1}$ ) per well, diluted in coating buffer overnight at $4^{\circ} \mathrm{C}$.

9| Flick out the primary antibody solution and add $200 \mu \mathrm{l}$ of blocking solution per well. Incubate at room temperature for $2 \mathrm{~h}$. 
10| Wash all the wells three times with $250 \mu \mathrm{l}$ of washing buffer per well.

11| Add $50 \mu \mathrm{l}$ of secondary antibody per well (e.g., BAF201 at $60 \mathrm{ng} \mathrm{ml}^{-1}$ ) diluted in blocking solution and add $50 \mu \mathrm{l}$ of samples per well from whole-blood incubation (Step $4 \mathrm{~A}$ ) or $50 \mu \mathrm{l}$ of samples per well from the cryopreserved blood incubation (Step 4B). Incubate at room temperature for $2 \mathrm{~h}$.

$\triangle$ CRITICAL STEP Change pipette tips to avoid cross contamination.

12| Wash all the wells four times with $250 \mu$ washing buffer per well.

13| Add $100 \mu \mathrm{l}$ of $50 \mathrm{ng} \mathrm{ml}^{-1}$ streptavidin peroxidase per well. Incubate at room temperature for $30 \mathrm{~min}$.

14| Wash all the wells eight times with $250 \mu \mathrm{l}$ of washing buffer per well.

15| Add $100 \mu$ of TMB substrate per well.

I CAUTION TMB is flammable. Toxic by inhalation, in contact with skin and if swallowed. Do not inhale the vapor. Avoid contact with eyes, skin and clothing. Avoid prolonged or repeated exposure. Do not use if skin is cut or scratched. Wash thoroughly after handling. Use in a fume hood.

16| Allow substrate metabolism in the dark for 5-20 min at $15-30{ }^{\circ} \mathrm{C}$. Stop the reaction with $50 \mu \mathrm{l}$ of $1 \mathrm{M} \mathrm{H}_{2} \mathrm{SO}_{4}$ per well. I CAUTION H $\mathrm{H}_{2} \mathrm{SO}_{4}$ causes severe burns. Do not inhale the vapor. Avoid contact with eyes, skin and clothes. Avoid prolonged or repeated exposure. Use in a fume hood.

17| Cover with a plastic tape and measure the $0 D$ at $450 \mathrm{~nm}$ against a reference wavelength of $600-690 \mathrm{~nm}$. $?$ TROUBLESHOOTING

\section{Data analysis TIMING $\mathbf{3 0}$ min}

18| Calculate the mean and s.d. of the OD of the replicate values of all controls and samples. Use the nonparametric Dixon's test or Grubb's test for normally distributed samples to detect and exclude possible outliers among the replicates.

19| Compare the mean OD of the negative control to $0.10 \mathrm{D}$.

\section{$?$ TROUBLESHOOTING}

20| Divide the mean OD for $0.5 \mathrm{EU} \mathrm{ml}^{-1}$ endotoxin control by the mean OD of negative control. ? TROUBLESHOOTING

21. Compare the mean OD of the spiked samples with the mean $O D$ of $0.5 \mathrm{EU} \mathrm{ml}^{-1}$ endotoxin control.

\section{? TROUBLESHOOTING}

\section{TIMING}

Step 1, Incubation plan design: $15 \mathrm{~min}$

Step 2, Preparation of endotoxin controls: $15 \mathrm{~min}$

Step 3, Sample preparation: $30 \mathrm{~min}$

Steps 4-7, Whole blood incubation: 13-27 h

Steps 8-17, ELISA: $20 \mathrm{~h}$

Steps 18-21, Data analysis: $30 \mathrm{~min}$

\section{? TROUBLESHOOTING}

Troubleshooting advice can be found in Table 4 . 
TABLE 4 | Troubleshooting table.

\begin{tabular}{|c|c|c|c|}
\hline Step & Problem & Possible reason & Possible solution \\
\hline \multirow[t]{4}{*}{17} & $\begin{array}{l}\text { All wells turn equally yellow after } \\
\text { addition of acid }\end{array}$ & $\begin{array}{l}\text { Plate was coated with detection } \\
\text { antibody instead of capture antibody }\end{array}$ & Repeat Steps 8-17 with samples from Step 7 \\
\hline & $\begin{array}{l}\text { Endotoxin control wells } \\
\text { remain blank }\end{array}$ & $\begin{array}{l}\text { Endotoxin control stock was too old or } \\
\text { thawed and refrozen too often }\end{array}$ & $\begin{array}{l}\text { Repeat entire procedure with new endotoxin } \\
\text { control stock }\end{array}$ \\
\hline & $\begin{array}{l}\text { Systematically increasing } \\
\text { signals from left to right on } \\
\text { the ELISA plate }\end{array}$ & $\begin{array}{l}\text { Contamination of samples with IL-1 } \\
\text { from other samples, e.g., by not } \\
\text { changing pipette tips appropriately or } \\
\text { defective ELISA plate }\end{array}$ & $\begin{array}{l}\text { Repeat Steps 8-17 with samples from Step } 7 \text {, } \\
\text { changing the pipette tips appropriately or test } \\
\text { batch of ELISA plates by applying the same } \\
\text { sample throughout }\end{array}$ \\
\hline & All wells remain blank & $\begin{array}{l}\text { Detection antibody was not added or } \\
\text { destroyed by freezing and thawing }\end{array}$ & $\begin{array}{l}\text { Repeat Steps 8-17 with samples from Step } 7 \text { and } \\
\text { a new aliquot of detection antibody }\end{array}$ \\
\hline 19 & $\begin{array}{l}\text { Mean } O D \text { of the negative } \\
\text { control replicates }>0.1 O D\end{array}$ & $\begin{array}{l}\text { The blood was prestimulated (e.g., } \\
\text { infection, allergy) or the saline or RPMI } \\
1640 \text { was contaminated }\end{array}$ & $\begin{array}{l}\text { Repeat whole blood incubation with blood from } \\
\text { a different donor and a new tube of saline or } \\
\text { RPMI } 1640\end{array}$ \\
\hline 20 & 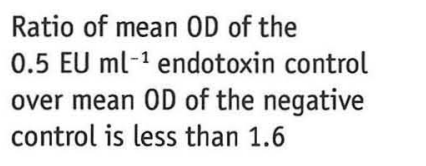 & $\begin{array}{l}\text { The endotoxin control was destroyed } \\
\text { by repeated freezing and thawing, is too } \\
\text { old or was set up incorrectly }\end{array}$ & $\begin{array}{l}\text { Repeat assay with a new aliquot of endotoxin } \\
\text { control }\end{array}$ \\
\hline 21 & Interference test is positive & $\begin{array}{l}\text { Samples may be lipidic, toxic or } \\
\text { immunomodulatory }\end{array}$ & $\begin{array}{l}\text { Repeat assay with higher dilution of sample. } \\
\text { Other adaptations of the assay may be } \\
\text { applicable } \\
21,30,31\end{array}$ \\
\hline
\end{tabular}

ELISA, enzyme-linked immunosorbent assay; OD, optical density.

\section{ANTICIPATED RESULTS}

Figure 2 shows an ELISA plate of an IPT carried out with fresh human whole-blood incubated for $20 \mathrm{~h}$ after stopping the TMB metabolism with sulfuric acid according to the incubation plan given in Table 1.

The minimum assay suitability requirements are as follows:

- The mean OD of control samples is 0.1 OD or below.

- The ratio of mean OD of $0.5 \mathrm{EU} \mathrm{ml}^{-1}$ endotoxin control over the mean OD of control samples is greater than 1.6.

- Interference of the samples is excluded. If an unspiked sample is nonpyrogenic, i.e., the OD lies below the mean OD of $0.5 \mathrm{EU}$ endotoxin control, and the mean $\mathrm{OD}$ of the respective spiked sample lies within a $50-200 \%$ range of the mean OD $\left(0.5 \mathrm{EU} \mathrm{ml}^{-1}\right)$, this sample is free of interference.

If the minimum assay suitability requirements are fulfilled, the prediction model can be applied: Rabbits are likely to develop fever if tested with $10 \mathrm{ml} \mathrm{kg}-1$ of the sample if: mean OD (sample) > mean OD $\left(0.5 \mathrm{EU} \mathrm{ml}^{-1}\right)$.

This is a qualitative evaluation for which the IPT has been validated, the readout being either "pyrogenic" or "nonpyrogenic". For a semi-quantitative assessment of the pyrogenic activity in liquid samples, the dilution which results in an $\mathrm{OD}$ that is comparable to $0.5 \mathrm{EU} \mathrm{ml}^{-1}$ endotoxin control can be used to calculate the endotoxin contamination in the undiluted sample. This estimation should be given in EEU because non-LPS pyrogens may also contribute to the pyrogenic activity. Similarly, the pyrogenic burden in air samples may be given in EEU by comparison of the IL-1 $\beta$ induction in the air monitor cassettes with that of $0.5 \mathrm{EU}$ $\mathrm{ml}^{-1}$ control. For solid samples a similar semi-quantitative assessment is possible if the samples can be employed in different quantities, e.g., fibers and beads. 0therwise only a rough estimation can be made by finding comparable IL-1 $\beta$

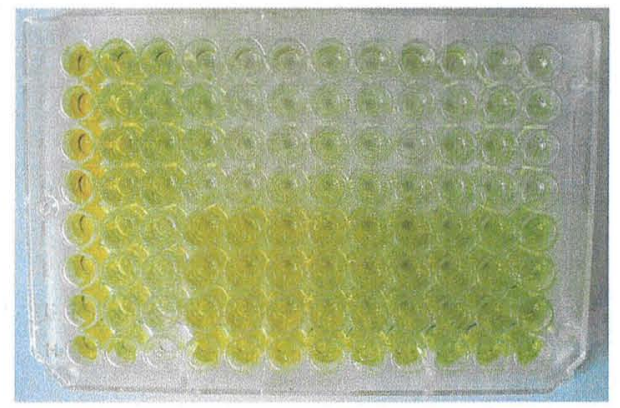

Figure 2 | Illustration of an enzyme-linked immunosorbent assay (ELISA) plate after the completion of an in vitro pyrogen test (IPT). ELISA plate of an IPT carried out with fresh human whole-blood incubated for $20 \mathrm{~h}$ after stopping the tetramethylbenzidine (TMB) metabolism with sulfuric acid. The plate setup conforms to the incubation plan given in Table 1. All experiments using human blood must comply with the legal requirements of your country and guidelines of your institution. 
Figure 3 | Analysis of pyrogenic activity of a cooling lubricant sample. (a) A cooling lubricant sample was employed in the in vitro pyrogen test (IPT) using fresh human whole blood in a series of dilutions (white bars). Spiked samples were prepared with $0.5 \mathrm{EU} \mathrm{ml}^{-1}$ lipopolysaccharides (LPS) (black bars). After $20 \mathrm{~h}$ incubation with whole blood the IL-1 $\beta$ enzymelinked immunosorbent assay (ELISA) was carried out and the absorbance at $450 \mathrm{~nm}$ recorded. Horizontal lines show the optical density (OD) of the 0.5 EU ml-1 endotoxin control as well as the range of $50-200 \%$ of this value. Error bars are the s.d. of quadruplicate samples. (b) After incubation the blood was subjected to a vitality assay (AlamarBlue metabolism) ${ }^{44}$. For comparison, fresh untreated human blood (vital) and ethanol (10\% vol/vol for $10 \mathrm{~min}$ )-treated human blood (dead) were employed in the assay. Error bars are the s.d. of quadruplicate samples. All experiments using human blood must comply with the legal requirements of the country and guidelines of the institution.

release in response to the sample and one of the endotoxin controls. This rough estimation would not consider the different shapes of concentration response curves from different types of pyrogens.

An example for the detection of pyrogenic activity in a liquid sample is given in Figure 3a. Here a cooling lubricant was tested for pyrogenic activity. Samples were employed at different dilutions and spiked with $0.5 \mathrm{EU} \mathrm{ml}^{-1}$ LPS at each of these dilutions. Horizontal lines show the $O D$ value of the endotoxin control $\left(0.5 \mathrm{EU} \mathrm{ml}^{-1}\right)$ and the range of $50-200 \%$ of this value. At dilutions below 1:100 interference is observed, as the spiked sample does not reach an $\mathrm{OD}$ greater than $50 \%$ of the endotoxin control. At dilutions starting from $1: 100$ the $O D$ of the spiked sample is within this range and interference is excluded. A vitality test (AlamarBlue metabolism) carried out with the diluted blood after the whole-blood incubation (Fig. $\mathbf{3 b}$ ) demonstrated that the interference at dilutions below 1:100 was caused by toxic effects to the blood cells.

Figure 4 shows an example of assessment of the pyrogenic burden in air samples from different environments. As would be expected, the pyrogenic burden in a hog house was far greater than that in an office environment.

In Figure 5 surgical steel beads were tested as an example of solid samples used in the IPT. The spiked sample results in an $0 \mathrm{D}$ in the $50-200 \%$ range of the $0.5 \mathrm{EU} \mathrm{ml}^{-1}$ endotoxin control, thus interference is excluded. The samples were negative for pyrogenic activity.

Different donors release different absolute amounts of IL-1 $\beta$ in response to pyrogenic stimulation. However, their sensitivity to pyrogens is highly comparable ${ }^{19}$. This is the reason why the endotoxin controls must always be run in parallel with the

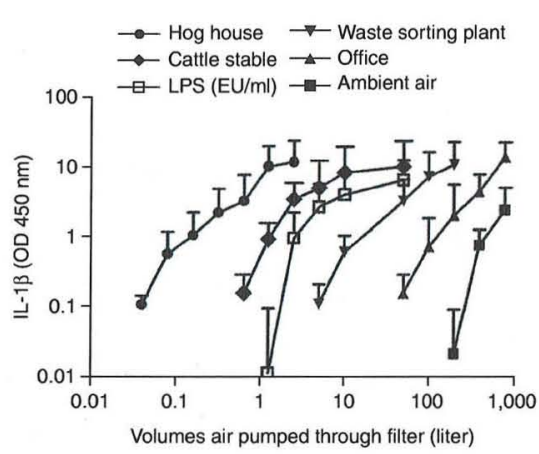

Figure 4 | Air quality assessment with the in vitro pyrogen test (IPT). Pyrogenic activity of particles from air sampled using air monitor cassettes was evaluated by IPT. Various volumes of air were sampled at different locations. The whole-blood incubation was carried out in air monitor cassettes with negative controls, endotoxin controls ( $\square$ ) (given in EU ml-1) and samples. After $20 \mathrm{~h}$ incubation with whole blood the IL-1 $\beta$ ELISA was carried out and the absorbance at $450 \mathrm{~nm}$ recorded. Error bars are the s.d. of quadruplicate samples. All experiments using human blood must comply with the legal requirements of the country and guidelines of the institution.

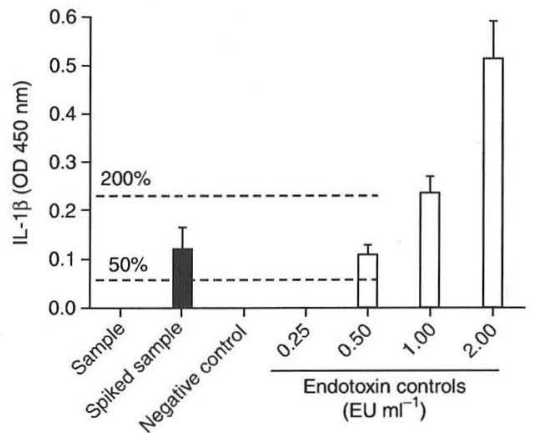

Figure 5 | Assessment of pyrogenic activity or interference of a steel sample by the in vitro pyrogen test (IPT). Samples of surgical steel beads were subjected to the IPT in $1.5 \mathrm{ml}$ reaction vials using fresh human whole blood. Parallel samples were spiked with $0.5 \mathrm{EU} \mathrm{ml}^{-1}$ lipopolysaccharides (LPS) for interference testing. After $20 \mathrm{~h}$ incubation with whole blood, the IL-1 $\beta$ enzyme-linked immunosorbent assay (ELISA) was carried out and the absorbance at $450 \mathrm{~nm}$ recorded. Horizontal bands show the range of $50-200 \%$ of the $0.5 \mathrm{EU} \mathrm{ml}^{-1}$ endotoxin control. All experiments using human blood must comply with the legal requirements of the country and guidelines of the institution. 
Figure 6 | Comparison of the response of fresh and cryopreserved human whole blood to different concentrations of lipopolysaccharides (LPS). Fresh and cryopreserved human whole blood from the same five donors was stimulated with the given concentrations of LPS. IL-1 $\beta$ was measured by enzyme-linked immunosorbent assay (ELISA). Error bars are the s.e. of the mean of the five donors' responses. All experiments using human blood must comply with the legal requirements of the country and guidelines of the institution.

sample assessment. Similarly, although the cryopreserved blood generally results in a somewhat higher absolute release of IL-1 $\beta$, the sensitivity of fresh blood and cryopreserved blood to pyrogens is highly comparable, see e.g., Figure 6.

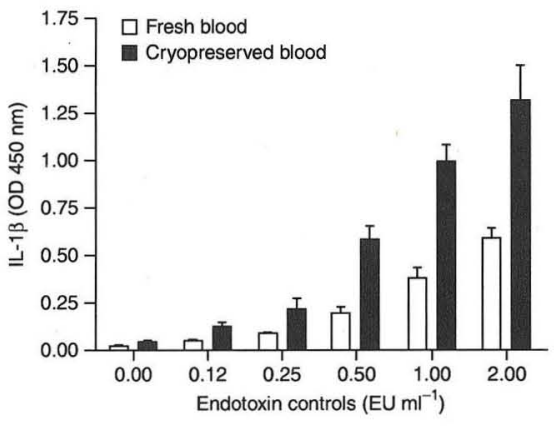

16. Li, S., Ballou, L.R., Morham, S.G. \& Blatteis, C.M. Cyclooxygenase-2 mediates the febrile response of mice to interleukin-1beta. Brain Res. 910, 163-173 (2001).

17. Li, S., Goorha, S., Ballou, L.R. \& Blatteis, C.M. Intracerebroventricular interleukin-6, macrophage inflammatory protein-1 beta and IL-18: pyrogenic and PGE(2)-mediated? Brain Res. 992, 76-84 (2003).

18. Hartung, T. \& Wendel, A. Detection of pyrogens using human whole blood. Altex 12, 70-75 (1995).

19. Wiegandt, M. Dissertation. Der Human Vollblut-Pyrogentest - Optimierung, Validierung und Vergleich mit den Arzneibuchmethoden. Heidelberg (2002).

20. Hoffmann, S. et al. International validation of novel pyrogen tests based on human monocytoid cells. J. Immunol. Methods 298, 161-173 (2005).

21. Schindler, S. et al. International validation of pyrogen tests based on cryopreserved human primary blood cells. J. Immunol. Methods $\mathbf{3 1 6}$ 42-51 (2006).

22. Wendel, A. \& Hartung, T. Test for determining pyrogenic effect of a material (DPC Biermann GmbH (Bad Nauheim, DE) Wendel, Albrecht (Tubingen, DE), Hartung, Thomas (Konstanz, DE), US Patent 5891728, 1999).

23. Wendel, A. \& Hartung, T. Use of frozen blood in a biological test method (DPC Biermann GmbH (Bad Nauheim, DE) Wendel, Albrecht (Tubingen, DE), Hartung, Thomas (Konstanz, DE), European Patent Ep0851231, 1997).

24. Hartung, T. Method for assaying flowing media for microbial toxins (Hartung, Thomas (Konstanz, DE) European Patent EP1377835, 2002).

25. Schindler, S. et al. Cryopreservation of human whole blood for pyrogenicity testing. J. Immunol. Methods 294, 89-100 (2004).

26. Mazzotti, F. et al. In vitro pyrogen test-a new test method for solid medical devices. J. Biomed. Mater Res. A 80, 276-282 (2007).

27. Hasiwa, M., Kullmann, K., von Aulock, S., Klein, C. \& Hartung, T. An in vitro pyrogen safety test for immune-stimulating components on surfaces. Biomaterials 28, 1367-1375 (2007).

28. Kindinger, I. et al. A new method to measure air-borne pyrogens based on human whole blood cytokine response. J. Immunol. Methods 298, 143-153 (2005).

29. Schindler, S. et al. Pyrogen testing of lipidic parenterals with a novel in vitro test-application of the IPT based on cryopreserved human whole blood. Pharmeur. Sci. Notes 2006, 1-7 (2006).

30. Daneshian, M., Wendel, A., Hartung, T. \& von Aulock, S. High sensitivity pyrogen testing in water and dialysis solutions. J. Immunol. Methods 336 64-70 (2008).

31. Daneshian, M., Guenther, A., Wendel, A., Hartung, T. \& von Aulock, S. In vitro pyrogen test for toxic or immunomodulatory drugs. J. Immunol. Methods 313, 169-175 (2006).

32. Welch, H., Calvery, H.O., McClosky, W.T. \& Price, W.T. Method of preparation and test for bacterial pyrogen. J. Am. Pharm. Assoc. 3, 65-69 (1943).

33. McClosky, W.T., Price, W.T., van Winkle, W.J., Welch, H. \& Calvery, H.O. Results of the first USP collaborative study of pyrogens. J. Am. Pharm. Assoc. 32, 69-73 (1943).

34. Grant, R. Emotional hypothermia in rabbits. Am. J. Physiol. 160, 285-290 (1950).

35. Hartung, T. et al. Novel pyrogen tests based on the human fever reaction. The report and recommendations of ECVAM Workshop 43. European Centre for the Validation of Alternative Methods. European Centre for the Validation of Alternative Methods. Altern. Lab. Anim. 29, 99-123 (2001).

36. Fennrich, S. et al. Detection of endotoxins and other pyrogens using human whole blood. Dev. Biol. Stand. 101, 131-139 (1999).

37. Dehus, 0., Hartung, T. \& Hermann, C. Endotoxin evaluation of eleven lipopolysaccharides by whole blood assay does not always correlate 
with limulus amebocyte lysate assay. J. Endotoxin Res. 12, 171-180 (2006).

38. Hartung, T. Statement on the validity of the in-vitro pyrogen test (European Centre for the Validation of Alternative Methods, Ispra, 2006)

39. Pott, G.B., Chan, E.D., Dinarello, C.A. \& Shapiro, L. Alpha-1-antitrypsin is an endogenous inhibitor of proinflammatory cytokine production in whole blood. J. Leukoc. Biol. 85, 886-895 (2009).

40. Poole, E.J., Dawson, P. \& Gaines Das, R.E. Second International standard for endotoxin: calibration in an international collaborative study. J. Endotoxin Res. 4, 221-231 (1997)
41. Dixon, W.J. Processing data for outliers. Biometrica 9, 74-89 (1953).

42. Grubbs, F.E. Sample criteria for testing outlying observations. Ann. Math. Stat. 21, 27-58 (1950).

43. Hoffmann, S., Luderitz-Puchel, U., Montag, T. \& Hartung, T. Optimisation of pyrogen testing in parenterals according to different pharmacopoeias by probabilistic modelling. J. Endotoxin Res. 11, 25-31 (2005).

44. Nakayama, G.R., Caton, M.C., Nova, M.P. \& Parandoosh, Z. Assessment of the Alamar Blue assay for cellular growth and viability in vitro. J. Immunol. Meth. 204, 205-208 (1997). 